KEK-TH-899

Jun. 2003

\title{
Effective Actions of Matrix Models on Homogeneous Spaces
}

\author{
Takaaki $\operatorname{IMAI}^{2) *}$, Yoshihisa KITAZAwA ${ }^{1), 2) \dagger}$ \\ Yastoshi TAKAYAMA ${ }^{2) \ddagger}$ AND Dan TOMINO ${ }^{1) \S}$ \\ 1) High Energy Accelerator Research Organization (KEK), \\ Tsukuba, Ibaraki 305-0801, Japan \\ 2) Department of Particle and Nuclear Physics, \\ The Graduate University for Advanced Studies, \\ Tsukuba, Ibaraki 305-0801, Japan
}

\begin{abstract}
We evaluate the effective actions of supersymmetric matrix models on fuzzy $S^{2} \times S^{2}$ up to the two loop level. Remarkably it turns out to be a consistent solution of IIB matrix model. Based on the power counting and SUSY cancellation arguments, we can identify the 't Hooft coupling and large $N$ scaling behavior of the effective actions to all orders. In the large $N$ limit, the quantum corrections survive except in 2 dimensional limits. They are $O(N)$ and $O\left(N^{\frac{4}{3}}\right)$ for 4 and 6 dimensional spaces respectively. We argue that quantum effects single out 4 dimensionality among fuzzy homogeneous spaces.
\end{abstract}

\footnotetext{
*e-mail address : imaitakaaki@yahoo.co.jp

${ }^{\dagger}$ e-mail address : kitazawa@post.kek.jp

$\ddagger$ e-mail address : takaya@post.kek.jp

$\S$ e-mail address : dan@post.kek.jp
} 


\section{Introduction}

Although string theory promises to tame quantum fluctuations of space-time, it also created deep questions of its own. One of such questions is to explain 4 dimensionality of space-time since the fundamental dimension in string theory is rather 10 or 11 . Traditionally invisible dimensions are assumed to be compactified at Planck scale. More recently, it has been recognized that we could as well be living on 4 dimensional branes with large or even infinite extra dimensions.

In any case, we certainly need to derive 4 dimensional gauge theory and gravitation from string theory. This problem can be compared to quark confinement problem in QCD. Any nonperturbative formulation of QCD must explain it. In this respect lattice gauge theory has been recognized as such since confinement is a natural phenomenon in the strong coupling limit.

We believe that matrix models are promising approach to investigate these nonperturbative questions in string theory [1][2]. Through them, string theory communicates with another promising idea of quantum space-time namely non-commutative geometry [3][4]. In fact, non-commutative(NC) gauge theory is naturally obtained in matrix models with noncommutative backgrounds [5][6]. The gauge invariant observables in NC gauge theory are the Wilson lines [7]. They play crucial roles to elucidate the gravitational aspects of NC gauge theory[8][9][10].

In the context of IIB matrix model, this question has been addressed from several different methods. We can cite branched polymer picture[11], complex phase effects[12] and mean-field approximations [13][14]. Although the results are encouraging thus far, it is fair to say that the problem is still far from settled. In particular the 4 dimensional gaussian distributions which have been obtained in the mean-field approximation are not realistic space-time yet.

In this respect, we find fuzzy homogeneous spaces to be more attractive. We have success-

fully constructed these spaces using IIB matrix models[18]. In the semiclassical limit, they reduce to Kähler manifolds up to dimension 6. Although we have not constructed experimentally favored de-Sitter space yet, they are closely related. Locally we obtain maximally supersymmetric Yang-Mills theory. Hence our model provides nonperturbative formulation of supersymmetric gauge theory as well if we can ignore gravitation [15][16].

In this paper, we investigate IIB matrix model with (and without) Myers terms[17]. By introducing a Myers term, we can construct non-commutative gauge theory on fuzzy sphere 
at classical level[19]. In our previous work, we have investigated quantum corrections of matrix models on fuzzy sphere up to the two loop level[20]. By modifying the Myers term, we can construct higher dimensional manifolds. In this paper, we investigate the simplest of such 4 dimensional manifolds, namely $S^{2} \times S^{2}$.

We compute the effective action up to the two loop level. Based on the power counting and SUSY cancellation arguments, we can identify the 't Hooft coupling and large $N$ scaling behavior of the effective actions to all orders. In the large $N$ limit, the quantum corrections survive except in 2 dimensional limits. They are generically $O(N)$ and $O\left(N^{\frac{4}{3}}\right)$ for 4 and 6 dimensional spaces respectively.

These fuzzy homogeneous spaces are possible backgrounds in IIB matrix model as well. Although they are not classical solutions, they may minimize the effective action at quantum level. With these motivations, we also compute the effective action of IIB matrix model without Myers term around such backgrounds. We indeed find that fuzzy $S^{2} \times S^{2}$ is a nontrivial solution of IIB matrix model at two loop level. In conjunction with our estimates of the large $N$ scaling behavior of the quatum corrections, we argue that 4 dimensionality is singled out among fuzzy homogeneous spaces.

The organization of this paper is as follows. In section 2, we investigate effective action of the deformed IIB matrix model whose classical solutions contain fuzzy $S^{2} \times S^{2}$. In section 3 , we investigate the effective actions for fuzzy $S^{2} \times S^{2}$ in IIB matrix model itself. We conclude in section 4 with discussions. In Appendices A and B, we explain detailed calculations of two loop effective actions on fuzzy $S^{2} \times S^{2}$ with and without a Myers term.

\section{Effective actions in matrix models}

NC gauge theories on compact homogeneous spaces $G / H$ can be constructed through matrix models. For this purpose, we may deform IIB matrix model as follows [18]

$$
S_{I I B} \rightarrow S_{I I B}+\frac{i}{3} f_{\mu \nu \rho} \operatorname{Tr}\left[A_{\mu}, A_{\nu}\right] A_{\rho}
$$

where $f_{\mu \nu \rho}$ is the structure constant of a compact Lie group $G$. Since there are 10 Hermitian matrices $A_{\mu}$ in IIB matrix model, the number of the Lie generators of $G$ cannot exceed 10 in this construction. Within such a constraint, we can realize fuzzy Kähler manifolds up to dimension 6 such as $S^{1}=S U(2) / U(1), C P^{2}=S U(3) / U(2)[21]$ or $C P^{3}=S O(5) / U(2)[22]$ as classical solutions of matrix models. 
Since these models possess the translation invariance

$$
A_{\mu} \rightarrow A_{\mu}+c_{\mu},
$$

and also

$$
\psi \rightarrow \psi+\epsilon
$$

we remove these zero-modes by restricting $A_{\mu}$ and $\psi$ to be traceless.

The equation of motion is

$$
\left[A_{\mu},\left[A_{\mu}, A_{\nu}\right]\right]+i f_{\mu \rho \nu}\left[A_{\mu}, A_{\rho}\right]=0 .
$$

The nontrivial classical solutions are

$$
A_{\alpha}^{c l}=t^{\alpha}, \quad \text { other } A_{\mu}^{c l}=0,
$$

where $t^{\alpha}$ 's satisfy the Lie algebra of $G$ or its sub-group. We have investigated quantum corrections in supersymmetric matrix models on fuzzy $S^{2}[20]$. In this paper we extend our investigations to higher dimensional fuzzy homogeneous space. Although we investigate the simplest of such manifolds: $S^{2} \times S^{2}$, it may reveal generic features of matrix models on homogeneous spaces.

In order to obtain NC gauge theory on fuzzy $S^{2} \times S^{2}$, we choose $G=S U(2) \times S U(2)$ with the following $f_{\mu \nu \rho}$ :

$$
\begin{aligned}
f_{\mu \nu \rho} & =f \epsilon_{\mu \nu \rho} ; \quad(\mu, \nu, \rho) \in(8,9,0), \\
f_{\mu \nu \rho} & =f \epsilon_{\mu \nu \rho} ; \quad(\mu, \nu, \rho) \in(1,2,3), \\
\text { other } f_{\mu \nu \rho}^{\prime} s & =0 .
\end{aligned}
$$

We investigate the following classical solutions:

$$
\begin{aligned}
A_{\mu}^{c l} & =f j_{\mu} \otimes 1 ; \quad(\mu=8,9,0), \\
A_{\mu}^{c l} & =f 1 \otimes \tilde{j}_{\mu} ; \quad(\mu=1,2,3), \\
\text { other } A_{\mu}^{c l} & =0
\end{aligned}
$$

where $j_{\mu}$ and $\tilde{j}_{\mu}$ are angular momentum operators. We further assume that $j_{\mu}$ and $\tilde{j}_{\mu}$ act on the $n$ copies of spin $l_{1}$ and $l_{2}$ representations respectively with $N=n\left(2 l_{1}+1\right)\left(2 l_{2}+1\right)$. In this paper, we always assume that $l_{i}$ are large which implies $n<<N$. These solutions 
represent $n$ coincident fuzzy $S^{2} \times S^{2}$. We refer to the constituent spaces as branes in this paper. In the limit $l_{1}=l_{2}$, our solution represents a 4 dimensional space while it reduces to a 2 dimensional space $\left(S^{2}\right)$ in the $l_{2}=0$ limit.

The reducibility implies that there are $n^{2}-1$ linearly independent Hermitian traceless matrices which commute with the classical solutions. They form the Lie algebra of $S U(n)$. Its Cartan subalgebra represents the relative center of mass coordinates of the branes. They will be called as zero-modes in what follows.

The classical action associated with this solution is

$$
-\frac{f^{4}}{6} N\left(l_{1}\left(l_{1}+1\right)+l_{2}\left(l_{2}+1\right)\right) \text {. }
$$

In the $4 \mathrm{~d}$ limit $\left(l_{1}=l_{2}\right)$, it becomes

$$
-\frac{f^{4}}{3} N l_{1}\left(l_{1}+1\right) \sim-\frac{f^{4}}{12 n} N^{2} .
$$

In the $2 \mathrm{~d}$ limit $\left(l_{2}=0\right)$, we obtain

$$
-\frac{f^{4}}{6} N l_{1}\left(l_{1}+1\right) \sim-\frac{f^{4}}{24 n^{2}} N^{3} .
$$

Since it is minimized when $l_{2}=0$ and $n=1$, a single fuzzy $S^{2}$ with $U(1)$ gauge group is classically favored. As for the reducible representations corresponding to the multiple branes, the classical action does not depend on their relative positions(zero-modes).

In our calculation of the partition function, we divide out the following gauge volume of $S U(N) / Z_{N}$ by gauge fixing

$$
2^{\frac{N^{2}+N}{2}-1} \pi^{\frac{N-1}{2}} \frac{1}{\sqrt{N}} \frac{1}{\prod_{k=1}^{N-1} k !}
$$

which appeared as the universal factor in [23]. We recall that the 'exact' free energy of IIB matrix model is as follows in this normalization[24]

$$
-\log \left(\sum_{n \mid N} \frac{1}{n^{2}}\right) .
$$

Let us denote the bosonic and fermionic zero-modes as $x_{\mu}$ and $\xi$. With the presence of zero-modes, we integrate massive modes first to obtain the Wilsonian effective action which is a functional of zero-modes. At the one loop level, we obtain[11]:

$$
\begin{aligned}
& \frac{1}{2} \operatorname{Tr} \log \left(P^{2} \delta_{\mu \nu}-2 i F_{\mu \nu}-2 i f_{\mu \nu \rho} P^{\rho}+\Xi \Gamma_{\mu} \frac{1}{\Gamma \cdot P} \Gamma_{\nu} \Xi\right) \\
& -\operatorname{Tr} \log \left(P^{2}\right)-\frac{1}{4} \operatorname{Tr} \log \left(\left(P^{2}+\frac{i}{2} F_{\mu \nu} \Gamma^{\mu \nu}\right)\left(\frac{1+\Gamma_{11}}{2}\right)\right),
\end{aligned}
$$


where $p_{\mu}=A_{\mu}^{c l}+x_{\mu}$ and

$$
\begin{aligned}
& {\left[p_{\mu}, X\right]=P_{\mu} X,} \\
& {\left[f_{\mu \nu}, X\right]=F_{\mu \nu} X, f_{\mu \nu}=i\left[p_{\mu}, p_{\nu}\right],} \\
& {[\xi, X]=\Xi X .}
\end{aligned}
$$

We first estimate (2.13) in the coincident limit where the bosonic zero-modes are small. Since the leading contributions in the large $N$ limit come from large eigenvalues, we expand (2.13) into the power series of $F_{\mu \nu}, P_{\mu}$ and $\Xi$. The leading contribution is

$$
\begin{gathered}
-2 \operatorname{Tr}\left[\frac{1}{P^{2}} P_{\alpha} \frac{1}{P^{2}} P_{\alpha}\right]-2 i \operatorname{Tr}\left[\frac{1}{P^{2}}\left[P_{\alpha}, P_{\beta}\right] \frac{1}{P^{2}} f_{\alpha \beta \gamma} P_{\gamma}\right] \\
=2 \operatorname{Tr} \frac{1}{P^{2}}=2 n^{2} \sum_{j p}(2 j+1)(2 p+1) \frac{1}{j(j+1)+p(p+1)} .
\end{gathered}
$$

In the $4 \mathrm{~d}$ limit $\left(l_{1}=l_{2}\right)$, we evaluate it as

$$
4 \log (2) n N \text {. }
$$

In the $2 \mathrm{~d}$ limit $\left(l_{2}=0\right)$, it becomes

$$
4 n^{2} \log (N / n) \text {. }
$$

In this process, we also obtain the products of the following polynomials which contain fermionic zero-modes:

$$
\begin{aligned}
& \operatorname{Tr} S^{m}, \\
& S_{\mu \nu}=\frac{1}{P^{2}} \bar{\Xi} \Gamma_{\mu} \frac{1}{\Gamma \cdot P} \Gamma_{\nu} \Xi,
\end{aligned}
$$

where $m \leq 8(n-1)$. Since these terms are less singular than (2.15) in the large $N$ limit, we may estimate $\operatorname{Tr} S^{m} \sim O\left(1 / f^{3 m}\right)$. After the fermion zero-mode integration, the normalization of the bosonic zero-mode integration measure is determines as

$$
\int d^{10(n-1)} x \frac{1}{f^{14(n-1)}(N / n)^{3(n-1)}} .
$$

In the presence of $n$ coincident branes, we have found that (2.16) and (2.17) scales as $n^{2}$ with $N / n$ being fixed. Since such configurations are further suppressed by the phase space of bosonic zero-modes, we conclude that the branes tend to move away from each other. 
If two branes are separated by a distance $x_{\mu}$ which is much larger than their radii $l$, we can approximate $\operatorname{Tr} S_{\mu \nu}$ as

$$
\operatorname{Tr} S_{\mu \nu} \sim \frac{(N / n)^{2}}{f^{3} x^{2}} \bar{\Xi} \Gamma_{\mu} \frac{1}{\Gamma \cdot x} \Gamma_{\nu} \Xi .
$$

After integrating fermion zero-modes, we obtain the following potential between them with respect to the identical bosonic zero-mode integration measure as in (2.19).

$$
\begin{aligned}
& 24 \log (|x| / l)+8 \log l \quad(2 d \text { limit }), \\
& 24 \log (|x| / l)-8 \log l \quad(4 d \text { limit }) .
\end{aligned}
$$

On the other hand, (2.15) can be estimated as

$$
-16 l^{2} \frac{1}{x^{2}}
$$

In the limit of $|x|>>l$, the former dominates the latter. Therefore the bosonic zero-mode integration converges at large distance and branes cannot move away from their neighbors much farther than their radii.

Let us consider loosely bound branched polymer like configurations of branes which are separated from their neighbors by a distance $d>>$. The one loop level effective action corresponding to such a configuration can be estimated as

$$
\begin{aligned}
& 16 \log (2) n l^{2}+(n-1) \log \left(f^{14} / l^{12}\right)+14(n-1) \log (d / l) \quad(4 d \text { limit }), \\
& 4 n \log (2 l)+(n-1) \log \left(f^{14} l\right)+14(n-1) \log (d / l) \quad(2 d \text { limit }) .
\end{aligned}
$$

This is the best upper bound of the one loop effective action we can obtain so far. We can interpret the first term in each limit as the one-loop self-energy of branes and the remaining terms as their interactions. We can trust the one loop estimation of brane interactions as long as they are well separated. Since the effective action is bounded by $O(n)$ quantity, it is consistent with our argument that $n$ coincident branes cannot overlap each other. We thus argue that $U(n)$ gauge symmetry is broken down to $U(1)$ at the one loop level.

We find it likely that branes settle into branched polymer like configurations by barely touching each other. ${ }^{5}$ From the both limits in (2.23), we observe that the one loop effective action favors the $2 \mathrm{~d}$ space $\left(S^{2}\right)$ over the $4 \mathrm{~d}$ space $\left(S^{2} \times S^{2}\right)$ just like the tree action.

\footnotetext{
${ }^{5}$ In the case of the $3 \mathrm{~d}$ model with two component Majorana spinor [19], the fermion zero-mode integration results in the vanishing partition function. Such an effect may suppress the formation of the branched polymer like configurations.
} 
We move on to study two loop corrections. Our strategy has been to obtain the Wilsonian effective action by integrating massive modes with fixed zero-modes. We delegate the detailed evaluation of the two loop effective actions to Appendix A. We first consider the $2 \mathrm{~d}$ limit $\left(l_{2}=0\right)$ which has been studied in [20]. The only novelty here is that we have the twice contribution from the Myers term (diagram (c) in Figure 1).

The two loop effective action with $U(n)$ gauge group is

$$
\begin{aligned}
F(l, 0) & =\frac{1}{f^{4}}\left(n^{3}\left(-40 F_{3}^{p}(l)+45 F_{5}(l)\right)-n\left(-40 F_{3}^{n p}(l)+45 F_{5}(l)\right)\right) \\
& \sim-35 \frac{1}{f^{4}(2 l+1)} n\left(n^{2}-1\right)+O\left(1 / N^{2}\right) .
\end{aligned}
$$

where

$$
\begin{aligned}
& F_{3}^{p}(l) \sim F_{3}^{n p}(l) \sim \frac{2.0}{2 l+1}, \\
& F_{5} \sim \frac{1}{(2 l+1)} .
\end{aligned}
$$

We summarize the effective action up to the two loop to the leading order of $1 / N$ as

$$
-\frac{f^{4}}{24 n^{2}} N^{3}+4 n^{2} \log (N / n)-35 \frac{1}{f^{4} N} n^{2}\left(n^{2}-1\right) .
$$

We still need to evaluate the higher order corrections beyond the two loop. Although we do not evaluate them explicitly in this paper, we can estimate their magnitude to all orders in perturbation theory based on our investigations thus far. In Feynman amplitudes of matrix models on $S^{2}$, the momentum integrations of field theory are replaced by finite series. Our important observation is that the large $N$ limit of the series can be estimated by the power counting arguments in field theory. It is well known that there is no ultraviolet divergences beyond the two loop level in $2 \mathrm{~d}$ gauge theory. We may then conclude that all series are convergent in the large $N$ limit in matrix models beyond two loop.

From this observation, we can estimate the $i$-th loop planar contribution to be $O\left(n^{i+1} /\left(f^{4} l\right)^{i-1}\right)$. It is because a single factor of $1 / l$ arises at each order due the $6 j$ symbols in the interaction vertices. It thus appears that the $i$-th loop contribution is $n^{2} O\left(\left(\lambda_{C M}^{2}\right)^{i-1}\right)$ where $\lambda_{C M}^{2}=4 \pi n^{2} /\left(f^{4} N\right)$ is the 't Hooft coupling of the commutative $U(n)$ gauge theory. In this way we can estimate the planar part of the effective action to all orders as:

$$
-\frac{\pi}{6 \lambda_{C M}^{2}} n N^{2}+4 n^{2} \log (N / n)+n^{2} h_{2}\left(\lambda_{C M}^{2}\right) .
$$

where $h_{2}\left(\lambda_{C M}^{2}\right)$ denotes a certain function of $\lambda_{C M}^{2}$. 
From these arguments, we find the effective action in the $2 \mathrm{~d}$ limit is $O\left(N^{2}\right)$ in the large $N$ limit with $\lambda_{C M}^{2}$ being kept fixed where the tree action dominates the quantum corrections. However the coincident limits may be exceptional configurations since we have argued that $U(n)$ gauge group is broken down to $U(1)$ by the dissociation process of branes at the one loop level.

In the case of a single brane with $U(1)$ gauge group, the two loop effective action is suppressed by another power of $1 / N$ :

$$
F(l, 0)=-40 \frac{1}{f^{4}}\left(F_{3}^{p}(l)-F_{3}^{n p}(l)\right) \sim-\frac{248}{f^{4} N^{2}} .
$$

It is because the two loop amplitude is convergent in our model and the theory becomes free (ordinary $U(1)$ gauge theory with adjoint matter) in the infrared limit. In other words, the planar and nonplanar contributions cancel to the leading order of $1 / N$. Since the gauge coupling of $\mathrm{NC} U(1)$ gauge theory is $\lambda^{2}=8 \pi / f^{4} N^{2}$, it is natural to find $O\left(\lambda^{2}\right)$ quantum corrections at two loop.

We can summarize the effective action up to the two loop to the leading order of $1 / N$ as

$$
-\frac{f^{4}}{24} N^{3}+4 \log (N)-\frac{248}{f^{4} N^{2}} .
$$

Since we expect the same pattern in higher orders, the structure of the effective action to all orders would be

$$
-\frac{\pi}{3 \lambda^{2}} N+4 \log (N)+h_{2}^{\prime}\left(\lambda^{2}\right)
$$

where $h_{2}^{\prime}$ denotes another function of $\lambda^{2}$.

In the absence of UV and IR contributions, we are left with supersymmetric $U(1) \mathrm{NC}$ gauge theory on $R^{2}$ in the large $N$ limit. The absence of $O(N)$ quantum corrections in (2.30) is consistent with the vanishing quantum corrections in the flat space. We thus argue that the effective action for a single brane is $O(N)$ with $\lambda^{2}$ being fixed. In such a large $N$ limit, the theory is again classical as the tree action dominates quantum corrections.

With the presence of $n$ branes, we have argued that the branched polymer like configurations with $U(1)$ gauge group are preferred at the one loop level. By assuming such configurations, the effective action for $n$ branes can be bounded from the above as before:

$$
\begin{aligned}
& -\frac{\pi}{3 \lambda^{2}} N+4 n \log (N / n)+n h_{2}^{\prime}\left(\lambda^{2}\right) \\
& -7(n-1) \log (\lambda)-6(n-1) \log (l)+14(n-1) \log (d / l),
\end{aligned}
$$


where $\lambda^{2} \sim n^{2} / f^{4} N^{2}$. The first and second lines in (2.31) correspond to the self-energy and interactions of branes respectively.

Since the classical action dominates quantum corrections in (2.31), a single brane minimizes it with $f^{4} N^{2}$ being fixed. Nevertheless the whole configurations with different numbers of branes energetically degenerate in the strong coupling limit. In such a situation, we argue that multi-brane configurations become dominant because of their large entropy.

We have pointed out that NC gauge theory on a fuzzy $S^{2}$ may be related to $2 \mathrm{~d}$ gravity[20]. It is well know that $2 \mathrm{~d}$ supergravity with the central charge $c>1$ is unstable against branched polymer formation. Since deformed IIB matrix model on $S^{2}$ with $U(1)$ gauge group naively corresponds to $c=8$, the formation of branched polymers in the strong coupling appears to be consistent with such a duality.

We next investigate the $4 \mathrm{~d}$ limit with $l_{1}=l_{2}$. Although the effective action could be quartically divergent by power counting, it is only quadratically divergent in this model. Since it is dominated by UV contributions, the effective action for $U(n)$ gauge group is well approximated by the planar contributions as

$$
-8 \frac{n^{3}}{f^{4}}\left(F_{3}^{p}(l, l)+2 F_{4}^{p}(l, l)\right) \sim-89.3 \frac{n^{3}}{f^{4}},
$$

where we have used the following numerically estimates:

$$
\begin{aligned}
& F_{3}^{p}(l, l)=3.24+O(1 / l), \\
& F_{4}^{p}(l, l)=3.96+O(1 / l) .
\end{aligned}
$$

We can simply put $n=1$ in (2.32) for $U(1)$ gauge group in contrast to the $2 \mathrm{~d}$ limit.

The total effective action up to the leading order of $1 / N$ is

$$
-\frac{f^{4}}{12 n} N^{2}+4 \log (2) n N-89.3 \frac{n^{3}}{f^{4}} .
$$

We can estimate the $i$-th loop contribution to be $\left.O\left(n^{i+1} l^{2} /\left(f^{4} l^{2}\right)^{i-1}\right)\right)$ since we obtain a factor of $1 / l^{2}$ from $6 j$ symbols in the interaction vertices. Here we also assume that the leading contributions cancel due to SUSY. Under the assumption, the amplitude is quadratically divergent which results in an over all factor of $l^{2}$. It thus appears that the $i$-th loop contribution is $O\left(n N\left(\lambda^{2}\right)^{i-1}\right)$ where $\lambda^{2}=(4 \pi)^{2} n^{2} /\left(f^{4} N\right)$ is the 't Hooft coupling of $U(n)$ gauge theory. In this way we can estimate the planar part of the effective action to all orders as:

$$
\left(-\frac{(2 \pi)^{2}}{3 \lambda^{2}}+4 \log (2)+h_{4}\left(\lambda^{2}\right)\right) n N .
$$


where $h_{4}\left(\lambda^{2}\right)$ denotes a function of $\lambda^{2}$. Therefore we find that the $4 \mathrm{~d}$ effective action is $O(N)$ in the large $N$ limit with $\lambda^{2}$ being fixed.

The effective action of loosely bound branched polymer like branes can be estimated as follows

$$
\begin{aligned}
& \left(-\frac{(2 \pi)^{2}}{3 \lambda^{2}}+4 \log (2)+h_{4}\left(\lambda^{2}\right)\right) N \\
& -7(n-1) \log (\lambda)-19(n-1) \log (l)+14(n-1) \log (d / l)
\end{aligned}
$$

where $\lambda^{2}=(4 \pi)^{2} n /\left(f^{4} N\right)$. Here again the first and second line correspond to the selfenergy and the interactions of branes respectively. We observe that the interactions are sub-dominant in comparison to the self-energy since the former is at most $O(n \log (l))$. Thus the favored $4 \mathrm{~d}$ configuration is such that it minimizes the self-energy with $f^{4} N$ being fixed. Since 't Hooft coupling $\lambda$ changes with $n$, we need to determine $h_{4}\left(\lambda^{2}\right)$ before answering such a question. We will estimate the strong coupling behavior of $h_{4}\left(\lambda^{2}\right)$ in the next section.

Nevertheless in the large $N$ scaling region with $f^{4} N$ fixed which is appropriate in $4 \mathrm{~d}$ limit, the effective action is negative and scales as $N^{2}$ in $2 \mathrm{~d}$ limit . Therefore in such a weak coupling regime, a single fuzzy $S^{2}$ always dominates in this model. The situation will be different in IIB matrix model which will be investigated in the next section.

We can further determine the large $N$ scaling behavior of the effective action in 6 d case $\left(S^{2} \times S^{2} \times S^{2}\right)$ in an analogous way. Such a space can be obtained as a classical solution of a matrix model by modifying the Myers term. The free energy with $U(n)$ gauge group is estimated as

$$
-a f^{4} N l^{2}+b n^{2} l^{4}-c \frac{n^{3} l^{6}}{f^{4} l^{3}}+\cdots,
$$

where $N=n(2 l+1)^{3}$. $a, b, c$ are calculable numerical coefficients. The $i$-th loop contribution can be estimated by power counting arguments as $O\left(n^{i+1} l^{4} /\left(f^{4}\right)^{i-1} l^{i-1}\right)$. We have also assumed here that the the leading contributions cancel due to SUSY in this model. Therefore the loop expansion may make sense if we fix $\lambda^{2}=n / f^{4} l$. In this way we can estimate the effective action to all orders as:

$$
\left(-\frac{a}{\lambda^{2}}+b+h_{6}\left(\lambda^{2}\right)\right) n^{2} l^{4}
$$

where $h_{6}\left(\lambda^{2}\right)$ denotes another function of $\lambda^{2}$. We conclude that the $6 \mathrm{~d}$ effective action is $O\left(N^{\frac{4}{3}}\right)$ in the large $N$ limit with $f^{4} N^{\frac{1}{3}}$ being fixed. 


\section{Effective action of IIB matrix model}

In this section, we investigate the effective action of IIB matrix model itself on fuzzy $S^{2} \times S^{2}$. Our investigation parallels with that in the previous section. The classical action of IIB matrix model for a configuration in $(2.7)$ is

$$
\begin{aligned}
& -\frac{1}{4} \operatorname{Tr}\left[A_{\mu}, A_{\nu}\right]\left[A_{\mu}, A_{\nu}\right] \\
= & \frac{f^{4}}{2} N\left(l_{1}\left(l_{1}+1\right)+l_{2}\left(l_{2}+1\right)\right),
\end{aligned}
$$

where $N=n\left(2 l_{1}+1\right)\left(2 l_{2}+1\right)$. If we fix $f$, the 4 dimensional limit $\left(S^{2} \times S^{2}\right)$ with $l_{1}=l_{2}$ is energetically favored over the two dimensional limit $\left(S^{2}\right)$ with $l_{2}=0$. We can also observe that larger gauge groups are favored. This preference is just opposite to the deformed IIB matrix model with the Myers term. However we still need to minimize the action (3.1) with respect to $f$. Since it is not stationary, we need to investigate higher order contributions to extract physical predictions.

The leading term of the one loop effective action in the large $N$ limit is

$$
\begin{aligned}
& -\operatorname{Tr}\left(\left({\frac{1}{P^{2}}}^{4}\right) F_{\mu \nu} F_{\nu \lambda} F_{\lambda \rho} F_{\rho \mu}\right)-2 \operatorname{Tr}\left(\left({\frac{1}{P^{2}}}^{4}\right) F_{\mu \nu} F_{\lambda \rho} F_{\mu \rho} F_{\lambda \nu}\right) \\
& +\frac{1}{2} \operatorname{Tr}\left(\left(\frac{1}{P^{2}}\right) F_{\mu \nu} F_{\mu \nu} F_{\lambda \rho} F_{\lambda \rho}\right)+\frac{1}{4} \operatorname{Tr}\left(\left({\frac{1}{P^{2}}}^{4}\right) F_{\mu \nu} F_{\lambda \rho} F_{\mu \nu} F_{\lambda \rho}\right) \\
= & 3 n^{2} \operatorname{Tr}\left(\frac{1}{P^{2}}\right)^{2}-6 n^{2} \operatorname{Tr} \frac{\left(P_{1}^{2}\right)^{2}+\left(P_{2}^{2}\right)^{2}}{\left(P^{2}\right)^{4}}+n^{2} \operatorname{Tr}\left(\frac{1}{P^{2}}\right)^{3} \\
= & 3 n^{2} \sum_{j, p} \frac{(2 j+1)(2 p+1)}{(j(j+1)+p(p+1))^{2}}-6 n^{2} \sum_{j, p} \frac{(2 j+1)(2 p+1)\left(j^{2}(j+1)^{2}+p^{2}(p+1)^{2}\right)}{(j(j+1)+p(p+1))^{4}} \\
& +n^{2} \sum_{j, p} \frac{(2 j+1)(2 p+1)}{(j(j+1)+p(p+1))^{3}} .
\end{aligned}
$$

In $2 \mathrm{~d}$ limit, it is estimated as

$$
-3 n^{2} \sum_{j} \frac{2 j+1}{(j(j+1))^{2}}+n^{2} \sum_{j} \frac{2 j+1}{(j(j+1))^{3}} \sim-2.6 n^{2} .
$$

In $4 \mathrm{~d}$ limit, we can estimate it as

$$
-n^{2} \log (N / n)
$$

The one loop corrections are found to be sub-leading since they are much smaller than those in the preceding section.

Our remaining task is to determine the structure of the effective actions to all orders. In $2 \mathrm{~d}$ limit, we can show that the tree action dominates over quantum corrections by repeating 
the same arguments in the previous section. It is because the Myers terms are soft in the sense that they do not alter power counting arguments. Since we can derive the identical long range interaction (2.21) as well, branes tend to form branched polymers. With fixed $f$, tree action favors larger $n$ in contrast to the preceding section. Hence the multiple brane configurations are always favored. The difference is that $f$ is now a parameter which describes the scale of field expectation values. We need to minimize the effective action with respect to $f$ as well. Since the tree action is not stationary, we may conclude that $2 \mathrm{~d}$ homogeneous spaces are after all not realized in IIB matrix model.

In $4 \mathrm{~d}$ case, we can repeat the same argument with the preceding section to show that the effective action is of the following form.

$$
\left(\frac{(2 \pi)^{2}}{\lambda^{2}}+\tilde{h}_{4}\left(\lambda^{2}\right)\right) n N
$$

where $\lambda^{2}=(4 \pi)^{2} n^{2} /\left(f^{4} N\right)$. $\tilde{h}_{4}\left(\lambda^{2}\right)$ is a function which describes the quantum corrections beyond one loop. Explicit evaluations of the two loop contribution in $4 \mathrm{~d}$ limit are reported in Appendix B. Our calculation shows that

$$
\tilde{h}_{4}\left(\lambda^{2}\right)=1.63 \frac{\lambda^{2}}{(2 \pi)^{2}}+\cdots .
$$

Our next task is to minimize the effective action not only with respect to $n$ but also with $f$ or equivalently $\lambda^{2}$. Due to the existence of nontrivial quantum corrections $\tilde{h}_{4}\left(\lambda^{2}\right), \lambda^{2}$ could be fixed as $\bar{\lambda}^{2}$ by minimizing (3.5) independently of $n$. At the two loop level, we find

$$
\frac{\bar{\lambda}^{2}}{(2 \pi)^{2}}=0.78
$$

where the effective action assumes the minimum: $2.55 n N$. Remarkably we find that $4 \mathrm{~d}$ homogeneous spaces $S^{2} \times S^{2}$ with $U(n)$ gauge group are solutions of IIB matrix model at two loop level as they minimize the effective action. Since the effective action is positive definite and $O\left(n^{2}\right)$, we further conclude that $U(1)$ gauge group is favored in the coincident limit. The discovery of a consistent solution representing a realistic 4 dimensional space-time in IIB matrix model is the most important result of this paper.

Although the existence of $4 \mathrm{~d}$ homogeneous spaces in IIB matrix model has been shown only at the two loop level, we can argue that higher order corrections do not destabilize it. It is because the effective action grows in the both weak and strong coupling directions as we will argue shortly. Since the effective action is believed to be bounded by (2.12), we may assume that $O(N)$ term is positive definite. With such an assumption, we can further 
conclude that a single $4 \mathrm{~d}$ homogeneous space $(n=1)$ minimizes the effective action in the coincident limit.

In the presence of $n$ identical $4 \mathrm{~d}$ branes, we still need to investigate their zero-modes. We find it likely that branched polymer like configurations with $U(1)$ gauge group will be favored again. It is because the effective action for such a configuration can be estimated as follows

$$
\left(\frac{(2 \pi)^{2}}{\bar{\lambda}^{2}}+\tilde{h}_{4}\left(\bar{\lambda}^{2}\right)\right) N
$$

where we have neglected the interaction terms which are at most $O(n \log (l))$. We thus find that the effective actions for different numbers of branes degenerate in $4 \mathrm{~d}$ limit to the leading order of $N$. This degeneracy could be lifted by the next leading $O(l \sim \sqrt{N})$ terms in the effective action. We have thus presented a scenario in which a single $4 \mathrm{~d}$ homogeneous space $S^{2} \times S^{2}$ with $U(1)$ gauge group is realized in IIB matrix model at quantum level. Although we hope to understand the dynamics of the zero-modes in more detail, it is beyond the scope of this paper.

In the remainder of this section, we argue that the homogeneous spaces are continuously connected to branched polymers. At the classical level, we have fuzzy homogeneous spaces made of $N$ quanta. The distance of the neighboring quanta is $O\left(f l^{\frac{1}{2}}\right)$. If we fix the coupling of NC gauge theory $g \sim 1 / f^{2} l$, the distance of the neighbors is $O\left(1 / g^{\frac{1}{2}}\right)$. If we fix $g \sim 1 / f^{2} l^{\frac{1}{2}}$ as in $6 \mathrm{~d}$ or $2 \mathrm{~d}$ with $U(n)$ gauge group, neighboring points are separated by distances of $O\left(l^{\frac{1}{4}} / g^{\frac{1}{2}}\right)$. They are homogeneously distributed on the respective manifolds.

We can investigate the quantum effects on the distribution of eigenvalues as follows. Let us introduce the norm of $A$ as $|A|^{2}=\operatorname{Tr} A A^{\dagger}$. We can also split $A=A^{c l}+a$ where $A^{c l}$ and $a$ denote the classical and quantum fields respectively.

In a single sphere case, we can obtain the following estimates up to the one loop level:

$$
\begin{aligned}
& \left|A^{c l}\right|=f l^{\frac{3}{2}}, \quad\left|a^{j}\right|=\frac{1}{f j} \\
& \frac{1}{N} \operatorname{Tr} A_{\mu} A_{\nu}=f^{2} l^{2} \bar{\delta}_{\mu \nu}+\frac{1}{f^{2} l} \log (l) \delta_{\mu \nu}
\end{aligned}
$$

where $\bar{\delta}_{\mu \nu}$ is projected in the sub-space in which $S^{2}$ resides.

In $4 \mathrm{~d}$ case,

$$
\begin{aligned}
& \left|A^{c l}\right|=f l^{2} n^{\frac{1}{2}}, \quad\left|a^{j, k}\right|=\frac{1}{f \sqrt{j^{2}+k^{2}}} \\
& \frac{1}{N} \operatorname{Tr} A_{\mu} A_{\nu}=f^{2} l^{2} \bar{\delta}_{\mu \nu}+\frac{n}{f^{2}} \delta_{\mu \nu}=\lambda N^{\frac{1}{2}}\left(\delta_{\mu \nu}+\frac{1}{\lambda^{2}} \bar{\delta}_{\mu \nu}\right),
\end{aligned}
$$


where $\bar{\delta}_{\mu \nu}$ assumes the value only in $S^{2} \times S^{2}$ sub-space.

In $6 \mathrm{~d}$ case,

$$
\begin{aligned}
& \left|A^{c l}\right|=f l^{\frac{5}{2}} n^{\frac{1}{2}}, \quad\left|a^{j, k, m}\right|=\frac{1}{f \sqrt{j^{2}+k^{2}+m}}, \\
& \frac{1}{N} \operatorname{Tr} A_{\mu} A_{\nu}=f^{2} l^{2} \bar{\delta}_{\mu \nu}+\frac{n l}{f^{2}} \delta_{\mu \nu}=\lambda N^{\frac{1}{2}}\left(\delta_{\mu \nu}+\frac{1}{\lambda^{2}} \bar{\delta}_{\mu \nu}\right),
\end{aligned}
$$

where $\bar{\delta}_{\mu \nu}$ assumes the value only in $S^{2} \times S^{2} \times S^{2}$ sub-space.

A naive extrapolation of these weak coupling expressions suggests the following correlation functions in the strong coupling limit for the both $4 \mathrm{~d}$ and $6 \mathrm{~d}$ cases:

$$
\frac{1}{N} \operatorname{Tr} A_{\mu} A_{\nu} \sim \lambda N^{\frac{1}{2}} \delta_{\mu \nu}
$$

In the strong coupling region, we can no longer hope to relate $\lambda$ to the classical field $A^{c l}$ as in the weak coupling region. $\lambda$ may be better defined as the parameter to describe the gauge invariant observables in (3.12).

The gauge invariant correlator in (3.12) measures the distribution of quanta. $\lambda N^{\frac{1}{2}}$ can be interpreted as their extension. This scaling behavior with respect to $N$ reminds us branched polymers whose fractal dimension is 4 [11]. For branched polymers of $N$ quanta which are separated by a distance $d$, the corresponding quantity is $(d N)^{\frac{1}{2}}$. The scaling behavior in (3.12) is consistent with the branched polymers whose $d \sim \lambda^{2}$.

The large $\lambda$ regime corresponds to the widely separated eigenvalue distribution of $A$. In such a regime, IIB matrix model is well approximated by the branched polymers and whose effective action can be estimated as

$$
14 N \log \left(\lambda^{2}\right)
$$

This is our prediction for the universal strong coupling behavior for the effective actions of IIB matrix model in homogeneous spaces. Our arguments here equally apply to the effective actions in the preceding section.

In $2 \mathrm{~d}$, the quantum fluctuation in (3.9) appear to be smaller than the classical field even with large $g \sim 1 / f^{2} l$ which is always smaller than $l$ in the large $N$ limit. However we have argued in section 2 that branched polymers with large numbers of constituents will be formed in the strong coupling limit which is consistent with (3.12).

In this section we have found that the effective action of IIB matrix model possesses the minimum with finite gauge coupling $\lambda$ in $4 \mathrm{~d}$ among the simplest fuzzy homogeneous spaces. 
The effective action favors 4 dimensionality since it is $O(N)$ in $4 \mathrm{~d}$ while it is $O\left(N^{\frac{4}{3}}\right)$ in $6 \mathrm{~d}$. On the other hand, it does not possess 2 dimensional solutions like $S^{2}$ since the theory is found to be classical in the large $N$ limit. What is significant in our observation is that quantum fluctuations naturally select 4 dimensionality. Furthermore homogeneous spaces may be smoothly connected with the branched polymers in the strong coupling limit.

\section{Conclusions and Discussions}

In this paper, we have investigated two loop effective actions of supersymmetric matrix models on a 4 dimensional fuzzy manifold $S^{2} \times S^{2}$. We find it remarkable that fuzzy $S^{2} \times S^{2}$ turns out be a nontrivial solution of IIB matrix model at two loop level. Based on the power counting and SUSY cancellation arguments, we have identified the 't Hooft coupling and large $N$ scaling behavior of the effective actions to all orders. In the large $N$ limit, the quantum corrections survive except in 2 dimensional limits. They are generically $O(N)$ and

$O\left(N^{\frac{4}{3}}\right)$ for 4 and 6 dimensional spaces respectively. These general arguments validate our solution beyond two loop level.

Although we have investigated the simplest 4 dimensional manifold, we believe our results capture generic feature of matrix models on homogeneous spaces. It is because we have employed power counting and SUSY cancellation arguments which do not depend on detailed group structures of homogeneous spaces. Therefore we believe that 4 dimensionality is a generic prediction of IIB matrix model among fuzzy homogeneous spaces.

Although 4 dimensional distributions are clearly favored in the mean field approximation, we believe we have made a substantial improvements from the optimization point of view as well. We have obtained $O(N)$ free energy while it is $O\left(N^{2}\right)$ in mean field approximation. However we still need to lower it down to the prediction in (2.12). We certainly hope to understand what kind of space-time achieves such a feat.

\section{Acknowledgments}

This work is supported in part by the Grant-in-Aid for Scientific Research from the Ministry of Education, Science and Culture of Japan. 


\section{Appendix A}

In this appendix, we evaluate the two loop effective action of NC gauge theory on $S^{2} \times S^{2}$. We consider NC gauge theory with $U(1)$ gauge group in the context of a deformed IIB matrix model with a Myers term. It is straightforward to generalize our results to the case of $U(n)$ gauge groups.

The evaluation procedure parallels to that of $\mathrm{NC}$ gauge theory on $S^{2}$. There are 5 diagrams to evaluate which are illustrated in Figure 1. (a),(b) and (c) represent contributions from gauge fields. (a) and (b) are of different topology while (c) involves the Myers type interaction. (d) involves ghost and (e) fermions respectively.

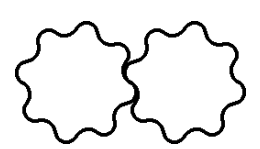

(a)

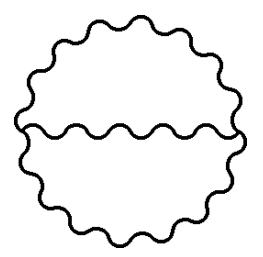

(b)

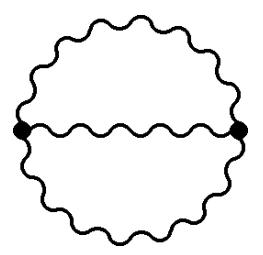

(c)

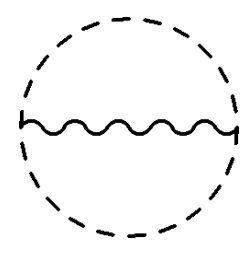

(d)

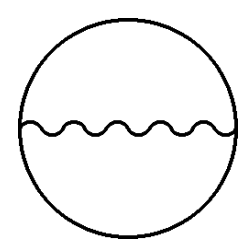

(e)

Figure 1: Feynman diagrams of 2 Loop corrections to the effective action

We expand matrices in terms of the tensor product of matrix spherical harmonics:

$$
\begin{aligned}
& A_{\mu}=f\left(p_{\mu}+\sum_{j m p q} a_{j m p q}^{\mu} Y_{j m} \otimes Y_{p q}\right) \\
& \psi=f^{\frac{3}{2}} \sum_{j m p q} \psi_{j m p q} Y_{j m} \otimes Y_{p q}
\end{aligned}
$$

where

$$
\begin{aligned}
& p_{\mu}=j_{\mu} \otimes 1 \quad(\mu=8,9,0), \\
& p_{\mu}=1 \otimes \tilde{j}_{\mu} \quad(\mu=1,2,3), \\
& \text { other } p_{\mu}^{\prime} s=0 .
\end{aligned}
$$

The summations over $j$ and $p$ run up to $j=2 l_{1}$ and $p=2 l_{2}$ respectively where we assume $N=\left(2 l_{1}+1\right) \times\left(2 l_{2}+1\right)$. We exclude the singlet state $j=p=0$ in the propagators in this paper. 
We adopt the following representation of $Y_{j m}$ :

$$
\left(Y_{j m}\right)_{s s^{\prime}}=(-1)^{l-s}\left(\begin{array}{ccc}
l & j & l \\
-s & m & s^{\prime}
\end{array}\right) \sqrt{2 j+1} .
$$

where they are normalized as

$$
\operatorname{Tr} Y_{j_{1} m_{1}} Y_{j_{2} m_{2}}=(-1)^{m_{1}} \delta_{j_{1}, j_{1}} \delta_{m_{1},-m_{2}}
$$

The cubic couplings of the matrix spherical harmonics can be evaluated as

$$
\begin{aligned}
& \operatorname{Tr}\left[Y_{j_{1} m_{1}} Y_{j_{2} m_{2}} Y_{j_{3} m_{3}}\right] \\
& =(-1)^{2 l} \sqrt{\left(2 j_{1}+1\right)\left(2 j_{2}+1\right)\left(2 j_{3}+1\right)} \\
& \times\left(\begin{array}{ccc}
j_{1} & j_{2} & j_{3} \\
m_{1} & m_{2} & m_{3}
\end{array}\right)\left\{\begin{array}{ccc}
j_{1} & j_{2} & j_{3} \\
l & l & l
\end{array}\right\} .
\end{aligned}
$$

We refer to $[25]$ for $(3 j)$ and $\{6 j\}$ symbols.

\section{bosonic propagators}

From the quadratic terms in the gauge fixed action, we can read propagators of gauge boson modes $a_{j m p q}^{\mu}$ and ghost modes $b_{j m p q}, c_{j m p q}$ as follows

$$
\begin{aligned}
\left\langle a_{j_{1} m_{1} p_{1} q_{1}}^{\mu} a_{j_{2} m_{2} p_{2} q_{2}}^{\nu}\right\rangle & =\frac{1}{f^{4}} \frac{(-1)^{m_{1}+q_{1}}}{j_{1}\left(j_{1}+1\right)+p_{1}\left(p_{1}+1\right)} \delta^{\mu \nu} \delta_{j_{1} j_{2}} \delta_{p_{1} p_{2}} \delta_{m_{1}-m_{2}} \delta_{q_{1}-q_{2}}, \\
\left\langle c_{j_{1} m_{1} p_{1} q_{1}} b_{j_{2} m_{2} p_{2} q_{2}}\right\rangle & =\frac{1}{f^{4}} \frac{(-1)^{m_{1}+q_{1}}}{j_{1}\left(j_{1}+1\right)+p_{1}\left(p_{1}+1\right)} \delta_{j_{1} j_{2}} \delta_{p_{1} p_{2}} \delta_{m_{1}-m_{2}} \delta_{q_{1}-q_{2}} .
\end{aligned}
$$

In terms of fields

$$
\begin{aligned}
a^{\mu} & =\sum_{j m p q} a_{j m p q}^{\mu} Y_{j m} \otimes Y_{p q}, \\
b & =\sum_{j m p q} b_{j m p q} Y_{j m} \otimes Y_{p q}, \\
c & =\sum_{j m p q} c_{j m p q} Y_{j m} \otimes Y_{p q}
\end{aligned}
$$

propagators become

$$
\begin{aligned}
\left\langle a^{\mu} a^{\nu}\right\rangle & =\frac{1}{f^{4}} \sum_{j m p q} \frac{(-1)^{m+q}}{j(j+1)+p(p+1)} \delta^{\mu \nu}\left(Y_{j m} \otimes Y_{p q}\right)\left(Y_{j-m} \otimes Y_{p-q}\right), \\
\langle c b\rangle & =\frac{1}{f^{4}} \sum_{j m p q} \frac{(-1)^{m+q}}{j(j+1)+p(p+1)}\left(Y_{j m} \otimes Y_{p q}\right)\left(Y_{j-m} \otimes Y_{p-q}\right) .
\end{aligned}
$$




\section{contribution from 4-gauge boson vertex (a)}

The interaction vertex which involves 4 gauge bosons is

$$
V_{4}=-\frac{f^{4}}{4} \operatorname{Tr}\left[a_{\mu}, a_{\nu}\right]\left[a_{\mu}, a_{\nu}\right] .
$$

It gives rises to the following contribution

$$
\begin{aligned}
< & -\frac{1}{1 !} V_{4}>1 \text { PI-2loop } \\
= & \frac{1}{4}\left(10^{2}-10\right) \frac{1}{f^{4}} \sum_{j_{1} j_{2}} \sum_{m_{1} m_{2}} \sum_{p_{1} p_{2}} \sum_{q_{1} q_{2}}(-)^{m_{1}+m_{2}+p_{1}+p_{2}} \\
& \times \frac{\operatorname{Tr}\left[Y_{j_{1} m_{1}} Y_{p_{1} q_{1}}, Y_{j_{2} m_{2}} Y_{p_{2} q_{2}}\right]\left[Y_{j_{1}-m_{1}} Y_{p_{1}-q_{1}}, Y_{j_{2}-m_{2}} Y_{p_{2}-q_{2}}\right]}{\left(j_{1}\left(j_{1}+1\right)+p_{1}\left(p_{1}+1\right)\right)\left(j_{2}\left(j_{2}+1\right)+p_{2}\left(p_{2}+1\right)\right)} .
\end{aligned}
$$

Here we can use the completeness condition:

$$
\sum_{j_{3} m_{3} p_{3} q_{3}}(-1)^{m_{3}+q_{3}}\left(Y_{j_{3} m_{3}} Y_{p_{3} q_{3}}\right)_{i j}\left(Y_{j_{3}-m_{3}} Y_{p_{3}-q_{3}}\right)_{k l}=\delta_{i l} \delta_{j k}
$$

We thus find

$$
\begin{aligned}
& -\frac{45}{2} \frac{1}{f^{4}} \sum_{j_{1} j_{2} j_{3}} \sum_{m_{1} m_{2}} \sum_{m_{3}} \sum_{p_{1} p_{2} p_{3}} \\
& \frac{\left(\operatorname{Tr} Y_{j_{3} m_{3}} Y_{p_{3} q_{3}}\left[Y_{j_{1} m_{1}} Y_{p_{1} q_{1}}, Y_{j_{2} m_{2}} Y_{p_{2} q_{2}}\right]\right)^{2}}{\left(j_{1}\left(j_{1}+1\right)+p_{1}\left(p_{1}+1\right)\right)\left(j_{2}\left(j_{2}+1\right)+p_{2}\left(p_{2}+1\right)\right)} \\
& =-45 \frac{1}{f^{4}} \sum_{j_{1} j_{2} j_{3}} \sum_{m_{1} m_{2}} \sum_{m_{3}} \sum_{p_{1} p_{2} p_{3}} \sum_{q_{1} q_{2} q_{3}} \\
& \frac{\left(2 j_{1}+1\right)\left(2 j_{2}+1\right)\left(2 j_{3}+1\right)\left(2 p_{1}+1\right)\left(2 p_{2}+1\right)\left(2 p_{3}+1\right)}{\left(j_{1}\left(j_{1}+1\right)+p_{1}\left(p_{1}+1\right)\right)\left(j_{2}\left(j_{2}+1\right)+p_{2}\left(p_{2}+1\right)\right)}\left(1-(-1)^{j_{1}+j_{2}+j_{3}+p_{1}+p_{2}+p_{3}}\right) \\
& \times\left(\begin{array}{ccc}
j_{1} & j_{2} & j_{3} \\
m_{1} & m_{2} & m_{3}
\end{array}\right)^{2}\left(\begin{array}{ccc}
p_{1} & p_{2} & p_{3} \\
q_{1} & q_{2} & q_{3}
\end{array}\right)^{2}\left\{\begin{array}{ccc}
j_{1} & j_{2} & j_{3} \\
l_{1} & l_{1} & l_{1}
\end{array}\right\}^{2}\left\{\begin{array}{ccc}
p_{1} & p_{2} & p_{3} \\
l_{2} & l_{2} & l_{2}
\end{array}\right\}^{2} \\
& =-45 \frac{1}{f^{4}}\left(F_{1}^{p}\left(l_{1}, l_{2}\right)-F_{1}^{n p}\left(l_{1}, l_{2}\right)\right) \text {, }
\end{aligned}
$$

where

$$
\begin{aligned}
& F_{1}^{p}\left(l_{1}, l_{2}\right)=\frac{1}{\left(2 l_{1}+1\right)\left(2 l_{2}+1\right)} \sum_{j_{1} j_{2}} \sum_{p_{1} p_{2}} \\
& \frac{\left(2 j_{1}+1\right)\left(2 j_{2}+1\right)\left(2 p_{1}+1\right)\left(2 p_{2}+1\right)}{\left(j_{1}\left(j_{1}+1\right)+p_{1}\left(p_{1}+1\right)\right)\left(j_{2}\left(j_{2}+1\right)+p_{2}\left(p_{2}+1\right)\right)}, \\
& F_{1}^{n p}\left(l_{1}, l_{2}\right)=\sum_{j_{1} j_{2}} \sum_{p_{1} p_{2}}(-1)^{j_{1}+j_{2}+p_{1}+p_{2}} \\
& \times \frac{\left(2 j_{1}+1\right)\left(2 j_{2}+1\right)\left(2 p_{1}+1\right)\left(2 p_{2}+1\right)}{\left(j_{1}\left(j_{1}+1\right)+p_{1}\left(p_{1}+1\right)\right)\left(j_{2}\left(j_{2}+1\right)+p_{2}\left(p_{2}+1\right)\right)}\left\{\begin{array}{ccc}
l_{1} & l_{1} & j_{2} \\
l_{1} & l_{1} & j_{1}
\end{array}\right\}\left\{\begin{array}{lll}
l_{2} & l_{2} & p_{2} \\
l_{2} & l_{2} & p_{1}
\end{array}\right\} .
\end{aligned}
$$


Here we have used the property of 3j-symbol:

$$
\sum_{m_{3}} \sum_{m_{1} m_{2}}\left(\begin{array}{ccc}
j_{1} & j_{2} & j_{3} \\
m_{1} & m_{2} & m_{3}
\end{array}\right)^{2}=\sum_{m_{3}} \frac{1}{2 j_{3}+1}=1,
$$

and the property of $6 \mathrm{j}$-symbol:

$$
\begin{aligned}
& \sum_{j_{1} p_{1}}\left(2 j_{1}+1\right)\left(2 p_{1}+1\right)\left\{\begin{array}{lll}
j_{1} & j_{2} & j_{3} \\
l_{1} & l_{1} & l_{1}
\end{array}\right\}^{2}\left\{\begin{array}{ccc}
p_{1} & p_{2} & p_{3} \\
l_{2} & l_{2} & l_{2}
\end{array}\right\}^{2} \\
= & \frac{1}{\left(2 l_{1}+1\right)\left(2 l_{2}+1\right)}, \\
& \sum_{j_{1} p_{1}}(-1)^{j_{1}+p_{1}}\left(2 j_{1}+1\right)\left(2 p_{1}+1\right)\left\{\begin{array}{lll}
j_{1} & j_{2} & j_{3} \\
l_{1} & l_{1} & l_{1}
\end{array}\right\}^{2}\left\{\begin{array}{ccc}
p_{1} & p_{2} & p_{3} \\
l_{2} & l_{2} & l_{2}
\end{array}\right\}^{2} \\
= & \left\{\begin{array}{lll}
l_{1} & l_{1} & j_{2} \\
l_{1} & l_{1} & j_{3}
\end{array}\right\}\left\{\begin{array}{lll}
l_{2} & l_{2} & p_{2} \\
l_{2} & l_{2} & p_{3}
\end{array}\right\} .
\end{aligned}
$$

\section{contribution from 3-gauge boson vertices (b)}

Firstly we calculate 2-loop 1PI contribution from 3-gauge boson vertices:

$$
V_{3}=\frac{f^{4}}{2} \operatorname{Tr}\left[p_{\mu}, a_{\nu}\right]\left[a_{\mu}, a_{\nu}\right]-\frac{f^{4}}{2} \operatorname{Tr}\left[p_{\nu}, a_{\mu}\right]\left[a_{\mu}, a_{\nu}\right] .
$$

The result can be expressed in a compact form as:

$$
\left\langle\frac{1}{2} V_{3} V_{3}\right\rangle=(10-1) \frac{1}{f^{4}}\left(<<\frac{P^{2}-P \cdot Q}{P^{2} Q^{2} R^{2}}>>_{p}-<<\frac{P^{2}-P \cdot Q}{P^{2} Q^{2} R^{2}}>>_{n p}\right),
$$

where $P, Q, R$ are defined as

$$
\begin{aligned}
P_{\mu} Y_{j_{1} m_{1}} Y_{p_{1} q_{1}} & \equiv\left[p_{\mu}, Y_{j_{1} m_{1}} Y_{p_{1} q_{1}}\right], \\
Q_{\mu} Y_{j_{2} m_{2}} Y_{p_{2} q_{2}} & \equiv\left[p_{\mu}, Y_{j_{2} m_{2}} Y_{p_{2} q_{2}}\right], \\
R_{\mu} Y_{j_{3} m_{3}} Y_{p_{3} q_{3}} & \equiv\left[p_{\mu}, Y_{j_{3} m_{3}} Y_{p_{3} q_{3}}\right] .
\end{aligned}
$$

We have also introduced the following average:

$$
\begin{aligned}
<<X>>_{p} & =\sum_{j_{i}, p_{i}, m_{i}, q_{i}} \Psi_{123}^{*} X \Psi_{123}, \\
<<X>>_{n p} & =\sum_{j_{i}, p_{i}, m_{i}, q_{i}} \Psi_{132}^{*} X \Psi_{123}, \\
\Psi_{123} & \equiv \operatorname{Tr} Y_{j_{1} m_{1}} Y_{j_{2} m_{2}} Y_{j_{3} m_{3}} \operatorname{Tr} Y_{p_{1} q_{1}} Y_{p_{2} q_{2}} Y_{p_{3} q_{3}} .
\end{aligned}
$$

Using the following relation,

$$
P \cdot Q \Psi_{123}=\frac{R^{2}-P^{2}-Q^{2}}{2} \Psi_{123},
$$


we evaluate (A.17) as

$$
\begin{aligned}
& \frac{27}{2 f^{4}}\left(<<\frac{1}{P^{2} Q^{2}}>>_{p}-<<\frac{1}{P^{2} Q^{2}}>_{n p}\right) \\
= & \frac{27}{2} \cdot \frac{1}{f^{4}} \sum_{j_{1} j_{2} j_{3}} \sum_{m_{1} m_{2} m_{3}} \sum_{p_{1} p_{2} p_{3}} \sum_{q_{1} q_{2} q_{3}}\left(1-(-1)^{\left.j_{1}+j_{2}+j_{3}+p_{1}+p_{2}+p_{3}\right)}\right. \\
& \times \frac{\left(2 j_{1}+1\right)\left(2 j_{2}+1\right)\left(2 j_{3}+1\right)\left(2 p_{1}+1\right)\left(2 p_{2}+1\right)\left(2 p_{3}+1\right)}{\left(j_{2}\left(j_{2}+1\right)+p_{2}\left(p_{2}+1\right)\right)\left(j_{3}\left(j_{3}+1\right)+p_{3}\left(p_{3}+1\right)\right)} \\
& \times\left(\begin{array}{ccc}
j_{1} & j_{2} & j_{3} \\
m_{1} & m_{2} & m_{3}
\end{array}\right)^{2}\left(\begin{array}{ccc}
p_{1} & p_{2} & p_{3} \\
q_{1} & q_{2} & q_{3}
\end{array}\right)^{2}\left\{\begin{array}{ccc}
j_{1} & j_{2} & j_{3} \\
l_{1} & l_{1} & l_{1}
\end{array}\right\}^{2}\left\{\begin{array}{ccc}
p_{1} & p_{2} & p_{3} \\
l_{2} & l_{2} & l_{2}
\end{array}\right\}^{2} \\
= & \frac{27}{2 f^{4}}\left(F_{1}^{p}\left(l_{1}, l_{2}\right)-F_{1}^{n p}\left(l_{1}, l_{2}\right)+F_{5}^{p}\left(l_{1}, l_{2}\right)-F_{5}^{n p}\left(l_{1}, l_{2}\right)\right)
\end{aligned}
$$

where

$$
F_{5}^{p}\left(l_{1}, l_{2}\right)=F_{5}^{n p}\left(l_{1}, l_{2}\right)=\frac{1}{\left(2 l_{1}+1\right)\left(2 l_{2}+1\right)} \sum_{j p \neq 00} \frac{(2 j+1)(2 p+1)}{(j(j+1)+p(p+1))^{2}} .
$$

Here we have used the following property of $6 \mathrm{j}$-symbol:

$$
\begin{aligned}
& \sum_{j_{1} p_{1} \neq 00}\left(2 j_{1}+1\right)\left(2 p_{1}+1\right)\left\{\begin{array}{lll}
j_{1} & j_{2} & j_{3} \\
l_{1} & l_{1} & l_{1}
\end{array}\right\}^{2}\left\{\begin{array}{ccc}
p_{1} & p_{2} & p_{3} \\
l_{2} & l_{2} & l_{2}
\end{array}\right\}^{2} \\
= & \frac{1}{\left(2 l_{1}+1\right)\left(2 l_{2}+1\right)}-\frac{\delta_{j 2, j 3} \delta_{p 2, p 3}}{\left(2 l_{1}+1\right)\left(2 l_{2}+1\right)\left(2 j_{2}+1\right)\left(2 p_{2}+1\right)}, \\
& \sum_{j_{1} p_{1} \neq 00}(-1)^{j_{1}+p_{1}}\left(2 j_{1}+1\right)\left(2 p_{1}+1\right)\left\{\begin{array}{lll}
j_{1} & j_{2} & j_{3} \\
l_{1} & l_{1} & l_{1}
\end{array}\right\}^{2}\left\{\begin{array}{ccc}
p_{1} & p_{2} & p_{3} \\
l_{2} & l_{2} & l_{2}
\end{array}\right\}^{2} \\
= & \left\{\begin{array}{lll}
l_{1} & l_{1} & j_{2} \\
l_{1} & l_{1} & j_{3}
\end{array}\right\}\left\{\begin{array}{lll}
l_{2} & l_{2} & p_{2} \\
l_{2} & l_{2} & p_{3}
\end{array}\right\}-\frac{\delta_{j 2, j 3} \delta_{p 2, p 3}}{\left(2 l_{1}+1\right)\left(2 l_{2}+1\right)\left(2 j_{2}+1\right)\left(2 p_{2}+1\right)} .
\end{aligned}
$$

It is because the singlet state is absent in the gluon propagator which results in the extra terms on the right-hand side of (A.23). Although they cancel each other in (A.21) for $U(1)$ case, it is not the case for $U(n)$ gauge groups.

\section{ghost contribution $(d)$}

We calculate the contribution from the ghost-gauge boson vertex:

$$
V_{g h}=-f^{4} \operatorname{Tr}\left[p_{\mu}, b\right]\left[c, a_{\mu}\right]
$$

The result is that:

$$
\frac{1}{f^{4}}\left(<<\frac{P \cdot Q}{P^{2} Q^{2} R^{2}}>>_{p}-<<\frac{P \cdot Q}{P^{2} Q^{2} R^{2}}>>_{n p}\right) .
$$




\section{contribution from cubic vertices (c)}

The cubic vertex which contains the structure constant of $S U(2) \times S U(2)$ is

$$
V_{c u b i c}=\frac{i}{3} f^{3} f_{\mu \nu \rho} \operatorname{Tr}\left[a_{\mu}, a_{\nu}\right] a_{\rho}
$$

Their contribution is

$$
\begin{aligned}
& <\frac{1}{2 !} V_{\text {cubic }} V_{\text {cubic }}>_{1 \text { PI-2loop }} \\
= & \frac{8}{f^{4}}\left(<<\frac{1}{P^{2} Q^{2} R^{2}}>>_{p}-<<\frac{1}{P^{2} Q^{2} R^{2}}>>_{n p}\right) \\
= & \frac{8}{f^{4}}\left(F_{3}^{p}\left(l_{1}, l_{2}\right)-F_{3}^{n p}\left(l_{1}, l_{2}\right)\right),
\end{aligned}
$$

where

$$
\begin{aligned}
F_{3}^{p}\left(l_{1}, l_{2}\right)= & \sum_{j_{1}, j_{2}, j_{3}} \sum_{p_{1}, p_{2}, p_{3}} \\
& \times \frac{\left(2 j_{1}+1\right)\left(2 j_{2}+1\right)\left(2 j_{3}+1\right)\left(2 p_{1}+1\right)\left(2 p_{2}+1\right)\left(2 p_{3}+1\right)}{\left(j_{1}\left(j_{1}+1\right)+p_{1}\left(p_{1}+1\right)\right)\left(j_{2}\left(j_{2}+1\right)+p_{2}\left(p_{2}+1\right)\right)\left(j_{3}\left(j_{3}+1\right)+p_{3}\left(p_{3}+1\right)\right)} \\
& \times\left\{\begin{array}{ccc}
j_{1} & j_{2} & j_{3} \\
l_{1} & l_{1} & l_{1}
\end{array}\right\}^{2}\left\{\begin{array}{ccc}
p_{1} & p_{2} & p_{3} \\
l_{2} & l_{2} & l_{2}
\end{array}\right\}^{2}, \\
F_{3}^{n p}\left(l_{1}, l_{2}\right)= & \sum_{j_{1}, j_{2}, j_{3} p_{1}, p_{2}, p_{3}}(-1)^{j_{1}+j_{2}+j_{3}+p_{1}+p_{2}+p_{3}} \\
& \times \frac{\left(2 j_{1}+1\right)\left(2 j_{2}+1\right)\left(2 j_{3}+1\right)\left(2 p_{1}+1\right)\left(2 p_{2}+1\right)\left(2 p_{3}+1\right)}{\left(j_{1}\left(j_{1}+1\right)+p_{1}\left(p_{1}+1\right)\right)\left(j_{2}\left(j_{2}+1\right)+p_{2}\left(p_{2}+1\right)\right)\left(j_{3}\left(j_{3}+1\right)+p_{3}\left(p_{3}+1\right)\right)} \\
& \times\left\{\begin{array}{lll}
j_{1} & j_{2} & j_{3} \\
l_{1} & l_{1} & l_{1}
\end{array}\right\}^{2}\left\{\begin{array}{lll}
p_{1} & p_{2} & p_{3} \\
l_{2} & l_{2} & l_{2}
\end{array}\right\}^{2} .
\end{aligned}
$$

\section{fermionic contribution (e)}

The fermion propagator is

$$
\begin{aligned}
-\frac{1}{f^{4}} \frac{1}{\Gamma^{\mu} P_{\mu}} & =\frac{1}{f^{4}} \frac{1}{P^{2}+\frac{i}{2} F_{\mu \nu} \Gamma^{\mu \nu}} \Gamma^{\rho} P_{\rho} \\
& =\frac{1}{f^{4}}\left(\frac{1}{P^{2}} \Gamma^{\mu} P_{\mu}+\frac{i}{2}\left(\frac{1}{P^{2}}\right)^{2} f_{\mu \nu \rho} \Gamma^{\mu \nu \sigma} P_{\rho} P_{\sigma}+O\left(\left(\frac{1}{P^{2}}\right)^{2}\right)\right) .
\end{aligned}
$$

We have expanded it in powers of $1 / P^{2}$ up to the first nontrivial order which is sufficient to calculate the leading contribution to the two loop effective action. 
2-loop (1PI) contribution to the effective action from the fermion-boson vertices is

$$
\begin{aligned}
& \frac{1}{f^{4}}\left(-64<<\frac{P \cdot Q}{P^{2} Q^{2} R^{2}}>_{p}+64<<\frac{\bar{P}^{2} \bar{Q}^{2}}{\left(P^{2}\right)^{2}\left(Q^{2}\right)^{2} R^{2}}>_{p}+64<<\frac{\bar{P} \cdot \bar{Q} \tilde{P} \cdot \tilde{Q}}{\left(P^{2}\right)^{2}\left(Q^{2}\right)^{2} R^{2}}>_{p}\right. \\
& \left.+64<<\frac{P \cdot Q}{P^{2} Q^{2} R^{2}}>>_{n p}-64<<\frac{\bar{P}^{2} \bar{Q}^{2}}{\left(P^{2}\right)^{2}\left(Q^{2}\right)^{2} R^{2}}>>_{n p}-64<<\frac{\bar{P} \cdot \bar{Q} \tilde{P} \cdot \tilde{Q}}{\left(P^{2}\right)^{2}\left(Q^{2}\right)^{2} R^{2}}>>_{n p}\right) \\
= & \frac{16}{f^{4}}\left[2 F_{1}^{p}\left(l_{1}, l_{2}\right)-2 F_{1}^{n p}\left(l_{1}, l_{2}\right)+F_{4}^{p}\left(l_{1}, l_{2}\right)-F_{4}^{n p}\left(l_{1}, l_{2}\right)\right] .
\end{aligned}
$$

where

$$
\begin{aligned}
& F_{4}^{p}\left(l_{1}, l_{2}\right) \\
& =\sum_{j_{1} j_{2} j_{3}} \sum_{p_{1} p_{2} p_{3}} \\
& \times \frac{\left(2 j_{1}+1\right)\left(2 j_{2}+1\right)\left(2 j_{3}+1\right)\left(2 p_{1}+1\right)\left(2 p_{2}+1\right)\left(2 p_{3}+1\right)}{\left(j_{1}\left(j_{1}+1\right)+p_{1}\left(p_{1}+1\right)\right)^{2}\left(j_{2}\left(j_{2}+1\right)+p_{2}\left(p_{2}+1\right)\right)^{2}\left(j_{3}\left(j_{3}+1\right)+p_{3}\left(p_{3}+1\right)\right)} \\
& \times\left(j_{3}\left(j_{3}+1\right) p_{3}\left(p_{3}+1\right)+2 j_{1}\left(j_{1}+1\right) p_{1}\left(p_{1}+1\right)-4 j_{3}\left(j_{3}+1\right) p_{1}\left(p_{1}+1\right)\right. \\
& \left.+2 j_{1}\left(j_{1}+1\right) p_{2}\left(p_{2}+1\right)+4 j_{1}\left(j_{1}+1\right) j_{2}\left(j_{2}+1\right)\right) \\
& \times\left\{\begin{array}{lll}
j_{1} & j_{2} & j_{3} \\
l_{1} & l_{1} & l_{1}
\end{array}\right\}^{2}\left\{\begin{array}{ccc}
p_{1} & p_{2} & p_{3} \\
l_{2} & l_{2} & l_{2}
\end{array}\right\}^{2}, \\
& F_{4}^{n p}\left(l_{1}, l_{2}\right) \\
& =\sum_{j_{1} j_{2} j_{3}} \sum_{p_{1} p_{2} p_{3}}(-1)^{j_{1}+j_{2}+j_{3}+p_{1}+p_{2}+p_{3}} \\
& \times \frac{\left(2 j_{1}+1\right)\left(2 j_{2}+1\right)\left(2 j_{3}+1\right)\left(2 p_{1}+1\right)\left(2 p_{2}+1\right)\left(2 p_{3}+1\right)}{\left(j_{1}\left(j_{1}+1\right)+p_{1}\left(p_{1}+1\right)\right)^{2}\left(j_{2}\left(j_{2}+1\right)+p_{2}\left(p_{2}+1\right)\right)^{2}\left(j_{3}\left(j_{3}+1\right)+p_{3}\left(p_{3}+1\right)\right)} \\
& \times\left(j_{3}\left(j_{3}+1\right) p_{3}\left(p_{3}+1\right)+2 j_{1}\left(j_{1}+1\right) p_{1}\left(p_{1}+1\right)-4 j_{3}\left(j_{3}+1\right) p_{1}\left(p_{1}+1\right)\right. \\
& \left.+2 j_{1}\left(j_{1}+1\right) p_{2}\left(p_{2}+1\right)+4 j_{1}\left(j_{1}+1\right) j_{2}\left(j_{2}+1\right)\right) \\
& \times\left\{\begin{array}{lll}
j_{1} & j_{2} & j_{3} \\
l_{1} & l_{1} & l_{1}
\end{array}\right\}^{2}\left\{\begin{array}{ccc}
p_{1} & p_{2} & p_{3} \\
l_{2} & l_{2} & l_{2}
\end{array}\right\}^{2} .
\end{aligned}
$$

\section{2-loop effective action}

In this way we find the total 2-loop free energy $F\left(l_{1}, l_{2}\right)$ of $U(1) \mathrm{NC}$ gauge theory on fuzzy $S^{2} \times S^{2}$ as follows

$$
-F\left(l_{1}, l_{2}\right)=8 \frac{1}{f^{4}}\left(F_{3}^{p}\left(l_{1}, l_{2}\right)-F_{3}^{n p}\left(l_{1}, l_{2}\right)+2 F_{4}^{p}\left(l_{1}, l_{2}\right)-2 F_{4}^{n p}\left(l_{1}, l_{2}\right)\right) .
$$

For $U(n)$ gauge group, we obtain

$$
-F\left(l_{1}, l_{2}\right)=\frac{1}{f^{4}}\left(n^{3}\left(8 F_{3}^{p}\left(l_{1}, l_{2}\right)+16 F_{4}^{p}\left(l_{1}, l_{2}\right)-45 F_{5}\left(l_{1}, l_{2}\right)\right)\right.
$$




$$
\left.+n\left(8 F_{3}^{n p}\left(l_{1}, l_{2}\right)+16 F_{4}^{n p}\left(l_{1}, l_{2}\right)-45 F_{5}\left(l_{1}, l_{2}\right)\right)\right) .
$$

We numerically find that planar contributions dominate over nonplanar contributions in $4 \mathrm{~d}$ limit.

$$
\begin{aligned}
F_{3}^{p}(l, l) & =3.24+O(1 / l), \\
F_{4}^{p}(l, l) & =3.96+O(1 / l), \\
F_{3}^{n p}(l, l) & =O(1 / l), \\
F_{4}^{n p}(l, l) & =O(1 / l), \\
F_{5}^{p}(l, l) & =F_{5}^{n p}(l, l)=O\left(\log (l) / l^{2}\right) .
\end{aligned}
$$

As the planar amplitudes are quadratically divergent, our findings are in accord with generic expectations in $\mathrm{NC}$ gauge theory.

\section{Appendix B}

We evaluate the two loop effective action of IIB matrix model in the $4 \mathrm{~d}$ limit in this appendix. The principle difference caused by the introduction of the Myers term resides in the gauge boson propagators. The inverse gauge boson propagator in IIB matrix model with $S^{2} \times S^{2}$ background is

$$
P^{2} \delta_{\mu \nu}+2 i f_{\mu \nu \rho} P^{\rho} .
$$

Since the leading contribution to the two loop effective action comes from the quadratically divergent part, we can approximate the propagators as follows:

$$
\delta_{\mu \nu} \frac{1}{P^{2}}-2 i f_{\mu \nu \rho} P^{\rho}\left(\frac{1}{P^{2}}\right)^{2}+4 I_{\mu \nu}(P)\left(\frac{1}{P^{2}}\right)^{3} .
$$

$I_{\mu \nu}(P)$ are the following transverse tensor in $S^{2} \times S^{2}$ :

$$
I_{\mu \nu}(P)=\bar{\delta}_{\mu \nu} \bar{P}^{2}-\bar{P}_{\mu} \bar{P}_{\nu}+\tilde{\delta}_{\mu \nu} \tilde{P}^{2}-\tilde{P}_{\mu} \tilde{P}_{\nu},
$$

where $\bar{P}_{\mu}$ and $\tilde{P}_{\mu}$ denote the components in the first and second three dimensional subspaces. The modification of the propagator is caused by a single or double insertions of $-2 i f_{\mu \nu \rho} P^{\rho} / P^{2}$ vertices into the minimal propagator.

Strictly speaking there is a zero mode in the gauge boson propagator which corresponds to the total angular momentum 2 in the both $S U(2)$. We may postpone to integrate this mode by considering the Wilsonian effective action. This problem does not modify the 
leading $O(N)$ term of the effective action since it arises from the quadratically divergent UV contributions as we shall find.

Since we do not have the Myers term in IIB matrix model, we should exclude the diagram (c). We also need to consider the effects caused by the modification of gauge field propagators in (B.2).

From diagram (a), we find the following extra planar contributions

$$
\begin{aligned}
& -\frac{72}{f^{4}} G_{1} \\
& -\frac{12}{f^{4}} G_{1}+\frac{6}{f^{4}} G_{2},
\end{aligned}
$$

where the first and the second line in (B.4) is due to the insertions of $-2 i f_{\mu \nu \rho} P^{\rho} / P^{2}$ vertices into the identical and different propagators respectively. We also have introduced the following functions.

$$
\begin{aligned}
G_{1}= & \frac{1}{N} \sum_{j_{1}, p_{1}, j_{2}, p_{2}}\left(2 j_{1}+1\right)\left(2 p_{1}+1\right)\left(2 j_{2}+1\right)\left(2 p_{2}+1\right) \\
& \times\left(\frac{1}{j_{1}\left(j_{1}+1\right)+p_{1}\left(p_{1}+1\right)}\right)^{2} \frac{1}{j_{2}\left(j_{2}+1\right)+p_{2}\left(p_{2}+1\right)}, \\
G_{2}= & <<\left(\frac{1}{P^{2}}\right)^{2}\left(\frac{1}{Q^{2}}\right)^{2} R^{2}>>_{p} .
\end{aligned}
$$

From (b) and (d), we find

$$
\begin{aligned}
& \frac{1}{f^{4}}\left(8 G_{1}^{\prime}+6 F_{3}+32 H_{1}-32 H_{2}\right) \\
& +\frac{1}{f^{4}}\left(-4 G_{1}^{\prime}+2 G_{2}+8 F_{3}-16 H_{3}+12 H_{4}-4 H_{5}\right),
\end{aligned}
$$

where the first and the second line in (B.6) is due to the insertions of $-2 i f_{\mu \nu \rho} P^{\rho} / P^{2}$ vertices into the identical and different propagators respectively as before. ${ }^{6}$

In this expression, we have introduced the following functions:

$$
\begin{aligned}
& G_{1}^{\prime}=<<\left(\frac{1}{P^{2}}\right)^{2} \frac{1}{Q^{2}}>>_{p}, \\
& H_{1}=<<P_{\mu} P_{\nu} I_{\mu \nu}(R)\left(\frac{1}{R^{2}}\right)^{3} \frac{1}{P^{2}} \frac{1}{Q^{2}}>>_{p},
\end{aligned}
$$

\footnotetext{
${ }^{6}$ In this evaluation, the following partial integration formula has been used.

$$
\sum_{m} P_{\mu} Y_{j m}^{\dagger} P_{\nu} Y_{j m}=-\sum_{m} Y_{j m}^{\dagger} P_{\nu} P_{\mu} Y_{j m}
$$




$$
\begin{aligned}
& H_{2}=<<P_{\mu} Q_{\nu} I_{\mu \nu}(R)\left(\frac{1}{R^{2}}\right)^{3} \frac{1}{P^{2}} \frac{1}{Q^{2}}>>_{p}, \\
& H_{3}=<<Q_{\mu} R_{\nu} I_{\mu \nu}(P)\left(\frac{1}{P^{2}}\right)^{2} \frac{1}{Q^{2}}\left(\frac{1}{R^{2}}\right)^{2}>>_{p}, \\
& H_{4}=<<Q_{\mu} Q_{\nu} I_{\mu \nu}(P)\left(\frac{1}{P^{2}}\right)^{2}\left(\frac{1}{Q^{2}}\right)^{2} \frac{1}{R^{2}}>>_{p}, \\
& H_{5}=<<Q_{\mu} R_{\nu} I_{\mu \nu}(P) \frac{1}{P^{2}}\left(\frac{1}{Q^{2}}\right)^{2}\left(\frac{1}{R^{2}}\right)^{2}>>_{p} .
\end{aligned}
$$

We can evaluate them as

$$
\begin{aligned}
G_{1}^{\prime}= & G_{1}+\frac{1}{N} \sum_{j, p} \frac{(2 j+1)(2 p+1)}{(j(j+1)+p(p+1))^{3}}, \\
G_{2}= & \sum_{j_{1}, j_{2}, j_{3}} \sum_{p_{1}, p_{2}, p_{3}}\left(2 j_{1}+1\right)\left(2 j_{2}+1\right)\left(2 j_{3}+1\right)\left(2 p_{1}+1\right)\left(2 p_{2}+1\right)\left(2 p_{3}+1\right) \\
& \times \frac{\left(j_{3}\left(j_{3}+1\right)+p_{3}\left(p_{3}+1\right)\right)}{\left(j_{1}\left(j_{1}+1\right)+p_{1}\left(p_{1}+1\right)\right)^{2}\left(j_{2}\left(j_{2}+1\right)+p_{2}\left(p_{2}+1\right)\right)^{2}} \\
& \times\left\{\begin{array}{lll}
j_{1} & j_{2} & j_{3} \\
l_{1} & l_{1} & l_{1}
\end{array}\right\}^{2}\left\{\begin{array}{ccc}
p_{1} & p_{2} & p_{3} \\
l_{2} & l_{2} & l_{2}
\end{array}\right\}^{2} .
\end{aligned}
$$

From (e)

$$
\begin{aligned}
& \frac{1}{f^{4}}\left(64 G_{1}^{\prime}-32 F_{3}+64 H_{2}\right) \\
& +\frac{1}{f^{4}}\left(32 G_{1}^{\prime}-16 G_{2}+64 H_{3}\right) .
\end{aligned}
$$

Here the first line in (B.10) is due to the double insertions of $-2 i f_{\mu \nu \rho} P^{\rho} / P^{2}$ vertices into the gauge boson propagator and the second line is due to the modifications of the both gauge boson and fermion propagators.

The total two loop effective action in IIB matrix model for $S^{2} \times S^{2}$ space is

$$
\begin{aligned}
-F & =\frac{1}{f^{4}}\left(16 G_{1}-8 G_{2}-18 F_{3}+32 H_{1}+32 H_{2}+48 H_{3}+12 H_{4}-4 H_{5}+16 F_{4}\right) \\
& =\frac{1}{f^{4}}\left(16 G_{1}-8 G_{2}-18 F_{3}+32 H_{3}+16 F_{4}\right),
\end{aligned}
$$

since $H_{1}+H_{2}=0, H_{3}+H_{4}=0$ and $H_{3}=H_{5} .{ }^{7}$ The only remaining independent function we need to evaluate is $H_{4}$ :

$$
\begin{aligned}
H_{4}= & \frac{1}{2} \sum_{j_{1}, j_{2}, j_{3}} \sum_{p_{1}, p_{2}, p_{3}} \frac{\left(2 j_{1}+1\right)\left(2 j_{2}+1\right)\left(2 j_{3}+1\right)\left(2 p_{1}+1\right)\left(2 p_{2}+1\right)\left(2 p_{3}+1\right)}{\left(j_{1}\left(j_{1}+1\right)+p_{1}\left(p_{1}+1\right)\right)^{2}\left(j_{2}\left(j_{2}+1\right)+p_{2}\left(p_{2}+1\right)\right)^{2}\left(j_{3}\left(j_{3}+1\right)+p_{3}\left(p_{3}+1\right)\right)} \\
& \times\left(-2\left(j_{1}\left(j_{1}+1\right)\right)^{2}-\left(j_{3}\left(j_{3}+1\right)\right)^{2}+2 j_{1}\left(j_{1}+1\right) j_{2}\left(j_{2}+1\right)+4 j_{1}\left(j_{1}+1\right) j_{3}\left(j_{3}+1\right)\right) \\
& \times\left\{\begin{array}{ccc}
j_{1} & j_{2} & j_{3} \\
l_{1} & l_{1} & l_{1}
\end{array}\right\}^{2}\left\{\begin{array}{ccc}
p_{1} & p_{2} & p_{3} \\
l_{2} & l_{2} & l_{2}
\end{array}\right\}^{2} .
\end{aligned}
$$

\footnotetext{
${ }^{7}$ We can further show that $F=2 F_{3} / f^{4}$.
} 
We have numerically estimated the following functions in the large $l$ limit as

$$
\begin{aligned}
& 2 G_{1}(l, l)-G_{2}(l, l)=1.93+O(1 / l), \\
& H_{4}(l, l)=0.822+O(1 / l), \\
& F(l, l)=\frac{1}{f^{4}} 6.53+O(1 / l) .
\end{aligned}
$$




\section{References}

[1] T. Banks, W. Fischler, S.H. Shenker and L. Susskind, M-theory as a matrix model: a conjecture, Phys. Rev. D55 5112 (1997), hep-th/9610043.

[2] N. Ishibashi, H. Kawai, Y. Kitazawa and A. Tsuchiya, A Large-N Reduced Model as Superstring, Nucl. Phys. B498 (1997) 467, hep-th/9612115.

[3] A. Connes, M. Douglas and A. Schwarz, Noncommutative geometry and matrix theory: compactification on tori, JHEP 9802: 003.1998, hep-th/9711162.

[4] N. Seiberg and E. Witten, String theory and non-commutative geometry, JHEP 9909 (1999) 032, hep-th/9908142.

[5] H. Aoki, N. Ishibashi, S. Iso, H. Kawai, Y. Kitazawa and T. Tada, Non-commutative Yang-Mills in IIB matrix model, Nucl. Phys. 565 (2000) 176, hep-th/9908141.

[6] M. Li, Strings from IIB Matrices, Nucl.Phys. B499 (1997) 149,hep-th/961222.

[7] N. Ishibashi, S. Iso, H. Kawai and Y. Kitazawa, Wilson loops in non-commutative YangMills, Nucl. Phys. B573 (2000) 573, hep-th/9910004.

[8] S. Minwalla, M.V. Raamsdonk and N. Seiberg, Non-commutative Perturbative Dynamics, JHEP 0002 (2000) 020,hep-th/9912072.

[9] D. J. Gross, A. Hashimoto and N. Itzhaki, Observables of Non-Commutative Gauge Theories, Adv.Theor.Math.Phys. 4 (2000) 893,hep-th/0008075.

[10] A. Dhar and Y. Kitazawa, Non-commutative Gauge Theory, Open Wilson Lines and Closed Strings, JHEP 0108 (2001) 044, hep-th/0106217.

[11] H. Aoki, S. Iso, H. Kawai, Y. Kitazawa and T. Tada, Space-Time Structures from IIB Matrix Model, Prog. Theor. Phys. 99, 713(1998); hep-th/9802085.

[12] Jun Nishimura, Graziano Vernizzi , SPONTANEOUS BREAKDOWN OF LORENTZ INVARIANCE IN IIB MATRIX MODEL JHEP 0004:015,2000: hep-th/0003223, K.N. Anagnostopoulos, J. Nishimura , NEW APPROACH TO THE COMPLEX ACTION PROBLEM AND ITS APPLICATION TO A NONPERTURBATIVE STUDY OF SUPERSTRING THEORY Phys.Rev.D66:106008: hep-th/0108041 
[13] J. Nishimura and F. Sugino, Dynamical Generation of Four-Dimensional Space-Time in IIB Matrix Model, JHEP 0205 (2002) 001, hep-th/0111102.

[14] H. Kawai, S. Kawamoto, T. Kuroki, T. Matsuo, S. Shinohara, Mean Field Approximation of IIB Matrix Model and Emergence of Four Dimensional Space-Time,Nucl.Phys. B647 (2002) 153-189: hep-th/0204240; Improved Perturbation Theory and FourDimensional Space-Time in IIB Matrix Model, Prog.Theor.Phys. 109 (2003) 115132:hep-th/0211272 .

[15] David B. Kaplan, Emmanuel Katz, Mithat Unsal, SUPERSYMMETRY ON A SPATIAL LATTICE, JHEP 0305:037,2003: hep-lat/0206019 ; Andrew G. Cohen , David B. Kaplan, Emanuel Katz, Mithat Unsal , SUPERSYMMETRY ON A EUCLIDEAN SPACE-TIME LATTICE. 1. A TARGET THEORY WITH FOUR SUPERCHARGES: hep-lat/0302017

[16] By Jun Nishimura , Soo-Jong Rey , Fumihiko Sugino , SUPERSYMETRY ON THE NONCOMMUTATIVE LATTICE, JHEP 0302:032,2003: hep-lat/0301025

[17] R.C. Myers,Dielectric-Branes, JHEP 9912 (1999) 022,hep-th/9910053.

[18] Y. Kitazawa, Matrix Models in Homogeneous Spaces, Nucl. Phys. B642 (2002) 210, hep-th/0207115.

[19] S. Iso, Y. Kimura, K. Tanaka and K. Wakatsuki, Non-commutative Gauge Theory on Fuzzy Sphere from Matrix Model, Nucl. Phys. B604 (2001) 121, hep-th/0101102.

[20] T. Imai, Y. Kitazawa, Y. Takayama and D. Tomino, Quantum Corrections on Fuzzy Sphere, Nucl. Phys. B665 (2003) 520, hep-th/0303120.

[21] D. Karabali and V.P. Nair, Quantum Hall Effect in Higher Dimensions, Nucl.Phys. B641 (2002) 533; hep-th/0203264.

[22] S.C. Zhang and J.P. Hu, A Four Dimensional Generalization of the Quantum Hall Effect, Science 294 (2001) 823; cond-mat/0110572.

[23] W. Krauth, H. Nicolai and M. Staudacher, Monte Carlo Approach to M-Theory, Phys. Lett. B431, 31 (1998); hep-th/9803117. 
[24] G. W. Moore, N. Nekrasov and S. Shatashvili, D-particle bound states and generalized instantons, Commun. Math. Phys. 209, 77 (2000); hep-th/9803265.

[25] A. R. Edmonds, Angular Momentum in Quantum Mechanics, Princeton Univ. Press (1957). 